\title{
Preanalytical issues identification and prevention in clinical hematology laboratory: best practice for patient safety
}

\section{Patel K. ${ }^{1}$}

${ }^{1}$ Dr. Kuntal Patel, Department of Pathology, Smt. B. K. Shah Medical Institute \& Research Centre, Sumandeep Vidyapeeth Deemed to be University, Vadodara, Gujarat, India.

Corresponding Author: Dr. Kuntal Devesh Patel, Department of Pathology, Central Laboratory, Dhiraj General Hospital, Sumandeep Vidyapeeth, Piparia, Waghodia Road, Vadodara, Gujarat. India, 391760. E-mail: kunta12789@gmail.com

\begin{abstract}
Introduction: Pre-analytical variable includes sample collection, transport, handling, physiological influences and/or interference factors. As the blood collection is the first step, any error in this step will endanger the whole test results, no matter how accurately these are analyzed in the laboratory. Material and Method: In present prospective study, some common issues in blood collection and handling in hospital inpatient and outdoor patient departments of Dhiraj General Hospital (tertiary health care center in Vadodara) have been discussed by giving some expected solutions. Pre-analytics involve the patient, the physician, the resident doctor, the nursing staff, the laboratory technician, the laboratory personnel and the transport service. Result: A total of 13178 received samples, the number of preanalytical issues documented were 185 in OPD and 388 in IPD. Conclusion: All the staff is required to know about preanalytical variables, their possible sources and their effect on the test results and ultimate outcome on patient's safety. Moreover, since the resident doctors have a direct interaction with the paramedical staff, it is very important for them to understand the preanalytical variables so that they could instruct the paramedical staff accordingly.
\end{abstract}

Keywords: Pre-analytical variables, Rejected samples, Acceptable sample, Hemolysed sample, Phlebotomy

\section{Introduction}

As current trend towards the consolidation of the clinical laboratories into mega structures and increased advanced automation; modern day diagnosis is profoundly dependent upon "reliable" laboratory data. Therefore, it is important to ensure credibility of the results originating from the clinical laboratories. There has been drastic improvement observed in sample collection, transport, dispatching of reports and in the best performance of these laboratories. Still there is long path to tread before $100 \%$ precision and accuracy can be achieved. Issues arising during sample processing are classified into preanalytical, analytical, and postanalytical, depending upon their source and time of presentation respectively. The pre-and post-analytical phases of the process account for $93 \%$ of errors [1].

All the processes occurring before the sample is processed in the autoanalyzer comprises in preanalytical phase; which include inappropriate tests that have been

Manuscript received: $16^{\text {th }}$ August 2019

Reviewed: $26^{\text {th }}$ August 2019

Author Corrected: $4^{\text {th }}$ September 2019

Accepted for Publication: $7^{\text {th }}$ September 2019 prescribed, improper sample collection \& transport and illegible handwriting on request forms. Because of these preanalytical errors, the labs have to bear the burden of the inconsistencies or incorrect reporting that can arise. Quality in laboratory medicine should be distinct as the assurance that each and every step in the total testing process (TTP) is appropriately executed, thus assuring valuable medical decision making and effective patient treatment. As stated by Lundberg several years ago on introducing the concept of the 'brain-to-brain loop' for describing the TTP, the generation of any laboratory test result involves nine steps: ordering, collection, identification, transportation, separation or preparation, analysis, reporting, and action [2].

Captivatingly, although the 'brain-to-brain' concept was defined as long as 30-40 years ago, it is still considered the working model in assuring quality and safety for requesting clinicians and patients. Certainly, subsequent changes made to the medical landscape have greatly impacted on the quality and delivery of laboratory services [3]. In the past decades, a ten-fold reduction in the analytical error rate has been achieved [4]. Thanks to improvements in the reliability and standardization of analytic techniques, reagents, and instrumentation, and advances in information technology, quality control 


\section{Original Research Article}

methods. During the past decade, after the publication of the Institute of Medicine (IOM) report, To Err Is Human, patient safety is finally the object of medical and public attention.

The awareness and understanding of medical errors have improved rapidly, with an bouncing patient safety movement promoting safer health care through 'systems' explanations, thanks to a major message from the IOM report: the cause of medical errors and preventable deaths was not careless or incompetent people but bad systems [5].

Compared with other types of medical error, however, errors in laboratory medicine received little attention and the reasons for this neglect are complex, as shown below:

- Heterogeneous and uncertain definition of what a laboratory error really is,

- Difficulties in identifying various types of errors and need for well-designed study protocols aiming to evaluate all steps in the total testing process (TTP);

- Complexity of TTP and need for cooperation and integration between different health-care providers;
- Poor perception by physicians and other nonlaboratory personnel of the harmfulness of errors in laboratory medicine

- Laboratory professionals disinclined to report and reveal data on types of errors and their frequency

- Increasing use of complementary/alternative testing options (e.g. point-of-care, near-patient and selfmonitoring).

In order to implement corrections and improve the testing process, the laboratory authorities must identify the various sources of errors. To ensure reliable test results and set the foundation for quality improvement, the current study was designed.

\section{Aims \& Objectives}

1. The aim is to analyze and enlist the prevalence of various preanalytical issues that has affected sample processing and ultimately patient's outcome.

2. To discuss solutions of preanalytical issues surfaced before sample processing in the clinical hematology laboratory.

\section{Method}

Dhiraj General Hospital (DGH) is a tertiary care center at Vadodara, Gujarat. It is a 1360-bedded super-specialty hospital offering specialized medical and surgical treatment to about 450000 patients including the outpatient department (OPD), emergency departments and in general and private wards every year.

This is prospective study and was performed in our clinical hematology laboratory during the period of three months from May 2019 to July 2019.

The clinical hematology laboratory is equipped with a contemporaneous autoanalyzer with Beckman coulter 750, SysmexKX21, Sysmex-RA 50, Stago STA compact, Vesmatic Easy for ESR, D-10 BioRad and other ancillaries for sample processing. Blood samples from outpatients are collected on site at a centralized collection center by laboratory personnel, whereas Inpatient phlebotomies are performed by clinical nursing staff. The venous samples were collected under proper sterile condition from anterior cubital fossa. The samples are delivered to the lab by the paramedical staff from the wards and laboratory support staff from the OPD respectively.

The samples were collected using Neecor tubes manually. Upon receiving the samples, the lab personnel visually detected any problems. When an error observed, entries are registered in the problem notification logbook. The data generated was reviewed on a weekly basis. The data collection procedure involved review of blood samples received from the inpatient as well as outpatient departments. The type of samples received were blood, urine, stool, CSF, fluids, semen and bone marrow.

Inclusion criteria: All the Hematological samples received to laboratory of tertiary care hospital for the period of the study duration.

Exclusion criteria: All the received samples other than hematological section. Data collection procedure and Data analysis: Venous blood samples were considered inappropriate according to the following definition of specimen types. (Table: 1)

Table-1: Definition of specimen types. 
Original Research Article

\begin{tabular}{|c|c|c|}
\hline 1 & Acceptable sample & - Without any interfering factor \\
\hline 2 & Inadequate sample & $\begin{array}{l}\text { - Sample not according to anticoagulant filled. } \\
\text { - Inappropriate volume (over/under filled sample) }\end{array}$ \\
\hline 3 & Clotted sample & $\begin{array}{l}\text { - Presence of visible clot/clots either red cell clot (EDTA) or fibrin clot } \\
\text { (citrate) }\end{array}$ \\
\hline 4 & Hemolysed sample & - Post centrifuged sample shows free hemoglobin in plasma $>100 \mathrm{mg} / \mathrm{L}$ \\
\hline 5 & Lipemic sample & $\begin{array}{l}\text { - Visible turbidity due to elevated concentrations of triglyceride level } \\
>1000 \mathrm{mg} / \mathrm{dL} \text { (whole blood) or }>300 \mathrm{mg} / \mathrm{dL} \text { (plasma) }\end{array}$ \\
\hline 6 & Icteric sample & - Visible detection of icterus is variable \\
\hline 7. & $\begin{array}{l}\text { Wrong } / \text { missing sample and } \\
\text { inadequate request form }\end{array}$ & $\begin{array}{l}\text { - Inappropriate container/wrong vials, } \\
\text { - Wrong/missing patient information, } \\
\text { - Lack of details/illegible handwriting on request form, } \\
\text { - Without request forms, } \\
\text { - Missing sample }\end{array}$ \\
\hline
\end{tabular}

Also, in DGH central lab, facility to outsource the samples to higher centers for special testing for which in-house facilities was not available.

The following Table: 2 of Preanalytical Variable were also evaluated while reporting included all criteria mentioned in Table: 1 for sample rejection.

Table-2: Preanalytical variables.

\begin{tabular}{|c|c|c|}
\hline Variables in specimen collection & Variables in specimen handling & Patient variable \\
\hline Fasting status & Requisition form & Age and Gender \\
\hline Presence of IV line & Transport condition & Medication \\
\hline Venous vs. capillary blood & Temperature & Smoking \\
\hline Anticoagulants and its ratio/tube filling error & Labeling & Pregnancy \\
\hline Order of draw & Hemolysis & Diet \\
\hline Tourniquet & Lipemia & Dehydration \\
\hline Time of collection & Centrifugation & Body mass \\
\hline Diurnal variation & Total Testing Process (TTP) time & Race \\
\hline Posture & Sunlight & . \\
\hline
\end{tabular}

The data were collected according to above mentioned criteria through pre-formed sheet and compiled with Microsoft Excel 2010. Patients consent was not needed as the study is observational and non-interventional.

\section{Result}

A total of 32507 samples from the outpatient department and in-house patients were received by our clinical hematology laboratory during the period of three months from May 2019 to July 2019. Out of these, 19329 samples were collected from the patients admitted in the wards and 13178 samples were collected in the outpatient department.

Here, first discussion is on the findings of the routine samples obtained from the outpatient department. A total of 13178 received samples, the number of preanalytical issues documented were 185 . This constitutes an error rate of $1.40 \%$. The distribution of the various preanalytical variables is depicted in Table 3.

Table-3: Frequency of pre-analytical issues observed in total 13178 OPD patients 


\begin{tabular}{|c|c|c|c|}
\hline Sr. No. & Variables & Frequency of issues & \% of issues \\
\hline A. & Acceptable samples & $\mathbf{1 2 9 9 3}$ & $\mathbf{9 8 . 6 0}$ \\
\hline B. & Rejected samples & $\mathbf{1 8 5}$ & $\mathbf{1 . 4 0}$ \\
\hline a & Inadequate sample & 32 & 0.25 \\
\hline b & Clotted sample & 13 & 0.10 \\
\hline c & Hemolysed sample & 39 & 0.30 \\
\hline d & Lipemic sample & 26 & 0.20 \\
\hline e & Icteric sample & 00 & 0.00 \\
\hline f & Wrong /missing sample and inadequate request form & 76 & 0.56 \\
\hline
\end{tabular}

The most frequent error encountered during processing was that of either wrong /missing samples or samples with inappropriate clinical data on request forms $(0.56 \%)$ followed by hemolysed sample $(0.30 \%)$.

Out of the 19329 blood collection tubes screened in IPD patients, issues were detected in 388 cases with $2.05 \%$ error rate over a period of 3 months. The distribution of the various preanalytical variables is represented in Table:4.

Table-4: Frequency of pre-analytical issues observed in total 19329 IPD patients.

\begin{tabular}{|c|c|c|c|}
\hline Sr. No. & Variables & Frequency of issues & \% of issues \\
\hline A. & Acceptable samples & $\mathbf{1 8 9 4 1}$ & $\mathbf{9 7 . 9 5}$ \\
\hline B. & Rejected samples & $\mathbf{3 8 8}$ & $\mathbf{2 . 0 5}$ \\
\hline a. & Inadequate sample & 57 & 0.30 \\
\hline b. & Clotted sample & 48 & 0.25 \\
\hline c. & Hemolysed sample & 149 & 1.30 \\
\hline d. & Lipemic sample & 38 & 0.20 \\
\hline e. & Icteric sample & 00 & 0.00 \\
\hline f. & Wrong /missing sample and inadequate request form & 96 & 0.50 \\
\hline
\end{tabular}

The results showed that hemolysis was responsible for majority of rejection in 149 samples, which accounts for $1.30 \%$ of the total number of samples received during this period.

A total of 96 samples were accompanied by inappropriate slips/ samples (ie, wrong requisition slip, without requisition slip, central registration number, clinical details, ward were not mentioned, missed/wrong samples). This comprised approximately $0.50 \%$ of all the samples received by the laboratory. Out of these 96 samples, laboratory personnel managed to ascertain correct patient data in 46 cases, and hence reporting was completed successfully for these patients, with extended turnaround time (TAT). Rest of samples could not be processed even after elaborate and painstaking efforts by the laboratory staff. Gross lipemia led to rejection of $0.20 \%$ in both IPD and OPD patients.

If all the errors are considered in a consolidated manner, then the total error rate for preanalytical variables in our lab was found to be $1.76 \%$.

\section{Discussion}

Recent advances in laboratory technology have led to many path-breaking innovations which have transformed cumbersome manual testing methods to fully automation, ensuring speed and accuracy. As laboratory cannot function in isolation. It is dependent upon other clinical departments, for properly filled request forms and samples for testing. Through the measly promotion of accuracy in the analytical phase of the testing process of the laboratory; reliability can't be achieved. The phases before the sample reaches the laboratory (preanalytical) and the phase after the sample is analyzed (post-analytical) are equally important [6]. 


\section{Original Research Article}

The preanalytical phase gets impacted with many shortcomings ranging from lax attitude about filling the requisition slips to the staff's lack of education about ideal phlebotomy procedures.

There has been varied information on the error rate within the whole lab testing procedure ( $0.1 \%$ to $9.3 \%)$. Plebani and Carraro observed in their paper that the great majority of errors result from problems in the preanalytical or post-analytical phases [7].The various variables leading to rejection of blood samples in the present study were discussed under different categories below:

A. Inadequate samples: Each hematological test process required a fixed volume of plasma for analysis. The main reasons behind this anomaly were ignorance of the phlebotomists, patients with chronic, debilitating diseases, and patients on chemotherapy, difficult sampling as in pediatric patients, whose thin veins were difficult to localize. Insufficient sample volume constituted the most frequent cause of test rejection in the samples collected in the OPD $(0.25 \%) \&$ in the IPD $(0.30 \%)$.

Centralized collection center where samples for clinical biochemistry, hematology and microbiology which were collected simultaneously were present. Due to the paucity of manpower, the ratio of patients to phlebotomists is unbalanced, making sample collection difficult. This may hinder accurate sample collection, leading to inadequate collection. One of the reasons for over/ under filled vials was patient rush.

The collection was carried out during fixed hours. Hence, this patient load combined with shortage of time may adversely affect proper sample collection in the OPD setting. Difficult sampling and patient non-compliance further exaggerated this problem. As a solution to this error is that it is obligatory for the laboratory staff to practice a certain basic level skillful phlebotomy technique to reduce such errors to a minimum.

B. Clotted sample: The receiving personnel have encountered clots while visual inspection of samples with error rate of $0.25 \%$ in IPD and $0.10 \%$ in OPD patients. As per our lab protocols, repeat samples were asked from ward side responsible staff. The reason for clotting of blood was inadequate mixing of samples, inappropriate ratio of anticoagulant to blood sample.

The sample to additive ratio is also very important e.g. for Prothrombin Time (PT) and for activated Partial Thromboplastin Time (aPTT). For these tests, blood was collected in citrated vial/tube in the ratio of 1:9 part of citrate and 9 parts of blood). If less blood was collected (e.g.1:7), then there was a significant increase in the aPTT results as compared to those which are obtained with the 1:9 ratio [8]. And if more blood was collected, there were chances of clot formation.

C. Hemolytic sample: Hemolysis was accounted for the majority of rejections in the present study. The frequency of hemolysis was more in the samples that were collected from the admitted patients as compared to the patients attending the OPDs ( $1.30 \%$ as compared to $0.30 \%)$. One reasonable explanation for this phenomenon could be the systematic blood collection technique followed by the laboratory staff in the OPD.

Hemolysis of samples occurs when blood is forced through a fine needle, shaking the tubes vigorously, high temperature and centrifuging the sample specimens before clotting was complete. Freezing and thawing of blood specimens may cause massive hemolysis [9]. In a study by Jay and colleagues, the majority of hemolysed samples (>95\%) could be attributed to in vitro processes resulting from incorrect sampling procedure or transportation [10]. Hemolysis leads to the extravasation of intracellular contents into the plasma, leading to interference with readings. It also leads to a prolonged turnaround time (TAT) due to the need for fresh samples for processing the request.

As a solution, vacutainers for plasma should be gently inverted a few times so the anticoagulant mixes with the blood. Also, regular in-house training sessions should be carried out for the technicians and bed side nursing staff to familiarize them with the standard protocols for sample processing. For this purpose, standard operating procedures (SOPs) were developed for the different steps involved in ideal laboratory operations and ethics.

Such training can facilitate in the adoption of ideal phlebotomy practices by sample collection team. The samples are thereby transported to our laboratory from the collection center by our staff following the basic precautions that must be adhered to during transportation.

There was an urgent need to instill awareness about the intricacies of a seemingly "simple and basic" activity that forms the mainstay of laboratory services - phlebotomy among the staff engaged in sample collection in our hospitals to effectively reduce inadvertent hemolysis.

The reason for incorrect phlebotomy practice included lack of awareness or possibly a heavy workload. This was the reason phlebotomy has been considered a separate area of improvement for medical technicians in developed countries. Those of us in developing nations must adopt a similar approach toward phlebotomy and 


\section{Original Research Article}

initiate steps for the inculcation of ideal phlebotomy practices among health care workers $[11,12]$.

A. Lipemic sample: Lipemia accounted for rejection of $0.20 \%$ of the samples in both inpatient and outpatient departments. Lipemic samples can arise due to collection after heavy meals or the presence of some metabolic disorder (hyperlipoproteinemias).

The solution to this was to avail sample only after an overnight fast. If the patient had a metabolic disorder, the same must be mentioned in the requisition slip. Lipemia interferes with optical reading by the instrument and can affect interpretation of ESR and coagulation profile values. A higher incidence of lipemia in OPD patients may be due to non-dissemination of information regarding prior preparation to the patients by the clinicians as well as non-compliance and/or miscomprehension of preparation rules by the patients. It is the responsibility of the clinicians and the phlebotomists to ensure that proper patient preparation is instituted before sample collection.

B. Icteric sample: Such samples didn't create any issue while the duration of present study.

\section{Wrong /missing sample and inadequate request}

form: A total of $0.56 \%$ samples in the wards were accompanied with these issues. The same figure for OPD samples was $0.50 \%$. It has been observed that the clinicians often send incomplete slips with the samples, which could be due to excessive patient load or lack of awareness regarding patient information. Modern day diagnostics was not only sample processing and preparation of reports. The laboratories were actively involved in disseminating information about critical results to clinicians for correlation of readings with clinical data. Incomplete/wrong patient information makes the practice redundant.

The laboratory staff could arrange the correct information about some of the patients admitted in the wards through their conscientious efforts. This led to the loss of precious time and is a labor-intensive activity; ultimately delaying of TAT. The same protocol could not be followed for the OPD patients as it was practically impossible to ascertain the patient/test information from either the clinicians or the patients. The approach towards the patient's request form was different. Patients with critical values were reached retrospectively. Those tests were repeated with fresh samples and new requisition slips as and when the patients revisited the hospital for checkup. This was definitely problematic for patients, who have to undergo the same process of registration and consequent sampling.
Such errors can be completely wiped out by sincere efforts of the clinicians to provide the same. This will facilitate speedy sample processing and report dispatch to the patients to initiate therapeutic interventions at the earliest. Carraro and Plebani performed a large comprehensive study that determined - of all errors detected- $8 . \%$ originated in the pre-analytic phase, compared with $18.5 \%$ in the post-analytic phase, and $13.3 \%$ during the analytic phase [7]. This group also found that, in more than $25 \%$ of all cases, the error resulted in unnecessary investigation or inappropriate patient care.

Some samples were found missing during manual transport. It has been found that samples were gone missing or went to other sections of central laboratory by helper staff who is not aware about the seriousness. The solution to this is to introduce pneumatic transport system (PTS).

From some sensitive wards like ICU, samples come to lab in bulk. So, receiving such a majority samples had made errors i.e. interchanging of samples, missed tests, wrong labeling etc. the solution to this is to introduce barcoding system for identification process of samples and reporting.

One important source of pre-analytical error is incorrect or incomplete information on the test request or labels which have been found in more than two thirds of all rejected samples in the laboratory [13].

Several other studies confirm that test requests can be a clinically important source of errors [14]. Paper based test requests are risky as they can be incompletely filled, placed in the wrong collection box, or simply be lost. Incomplete laboratory requests forms are rarely rejected at the service point and in many instances the reception staff in the laboratory may not know the significance of the missing data. Specific missing information included the physician's name, misidentification of patient and requested tests [15].

Labeling of specimen containers should always be done immediately before sample collection while, labeling them after sample collection increases the risk of the specimen collection from the wrong patient. Mislabeling is responsible for $50 \%$ of all identification errors [16]. Patient identification and wrong container labeling is probably the most important task in sample collection and error in this vital step could have mild to life threatening consequence. 
Original Research Article

Therefore, efforts to ensure amenability with standard identification procedures should be prioritized. The laboratory should establish rejection criteria and follow them closely. It is sometimes difficult to reject a sample, but it must be remembered that a poor sample will give poor results. The rejection criteria for minimizing errors must be set.

There is a need for stronger coordination between clinicians and personnel working outside the laboratory for improving the test quality. Clinical audits should be used as a tool to detect errors caused by organizational problems outside the laboratory.

\section{Conclusion}

Identifying the phase when the most errors occur enables laboratories to focus their quality improvement efforts. To conclude the study, pre-analytic error prevention requires excellent communication and cooperation among all members of the health care team, from the phlebotomist who collects the specimen, to the personnel handling the specimen.

Dependence on accurate laboratory results for establishing diagnosis makes it mandatory for labs to ensure accountability and accuracy of results and deny incorrect diagnosis due to faulty report. With close attention to established procedures and instructions, preanalytic error is minimized. In turn, patient care improves.

\section{What the study adds to the existing knowledge?}

Adoption of quality in all phases and not only the analytical processes and regular appraisal and audits is necessary to safeguard patient interests and deliver our services to society. Keeping record of the issues at all stages of analysis and formulating corrective strategies for their future prevention can progressively free a laboratory from such errors.

At the end, to summarize and taking into consideration the collected data of our set-up, following are the priority areas of patient safety improvement in laboratory practice, which is adding on the current knowledge of pre-analytical issues:

- Avoiding manual transcription of data;

- Appropriateness of test request;

- Sample collection and transport criteria;

- Sample acceptability and rejection criteria;

- Accuracy of patient / sample identification;
- Effectiveness of laboratory data communication including critical test results.

\section{Declarations}

Findings: Nil; Conflict of Interest: None initiated Permission from IRB: Yes

Acknowledgements: Author would like to express special thanks of gratitude to the Lab In-charge who gave the golden opportunity to do this wonderful project on the Pre analytical issues.

Secondly, author would also like to thank her husband \& her parents who helped in finalizing this project within the limited time frame.

Ethical Approval: ethical approval is not required about the content.

\section{References}

1. Leape, LL. Errors in medicine. Clin Chim Acta 2009; 404 (1):2-5. doi: 10.1016/j.cca.2009.03.020.

2.Adegoke OA, Idowu A, Jeje O. Incomplete laboratory request forms as a contributory factor to preanalytical errors in a Nigerian teaching hospital. Afr J Biochem Res. 2011;5(3):82-85.

3. Burnett L. Chesher D, Mudaliar Y. Improving the quality of information on pathology request forms. Ann Clin Biochem.2004;41(1):53-56. doi:https://doi.org/ 10. $1258 / 000456304322664708$

4. Lippi G, Blanckaert N, Bonini P, et al. Causes, consequences, detection and prevention of identification errors in laboratory diagnostics. Clin Chem Lab Med. 2009; 47 (2): 143-153. doi: 10. 1515/ CCLM. 2009. 045

5. Plebani M. Errors in clinical laboratories or errors in laboratory medicine? Clin Chem Lab Med. 2006;44 (6) : 750-759. doi: https://doi.org/10. 1515/ CCLM. 2006. 123

6. Lundberg GD. Acting on significant laboratory results. JAMA.1981;245(17):1762-1763.doi:10.1001 / jama. 1981.03310420052032

7. Carraro P. Hemolyzed specimens: A reason for rejection or a clinical challenge? Clin Chem. 2000; 46 (2) : 306-307.

8. Narayanan S, Hamasaki N. Current concepts of coagulation and fibrinolysis. Adv Clin Chem 1999;

Pathology Update: Tropical Journal of Pathology \& Microbiology Available online at: www.medresearch.in 625 | P a g e 
33:133-168.doi:https://doi.org/10.1016/S0065-2423(08) 60207-3

9. Jones BA, Calam RR, Howanitz PJ. Chemistry specimen acceptability. A College of American Pathologists Q-Probes study of 453 laboratories. Arch Pathol Lab Med. 1997;121(1):19-26.

10. Kirchner MJ, Funes VA, Adzet CB. Quality indicators and specifications for key processes in clinical laboratories: a preliminary experience. Clin Chem Lab Med 2007; 45(5):672-677. doi: https://doi. org/10. 1515/CCLM.2007.122

11. Jay DW, Provasek D. Characterization and mathematical correction of hemolysis interference in selected Hitachi 717 assays. Clin Chem. 1993;39(9): 1804-1810.

12. Fidler JR. Task analysis revisited: Refining the phlebotomy technician scope of practice and assessing longitudinal change in competencies. Eval Health Prof. 2007:30(2):150-169. doi: https://doi.org/10.1177/ 01632 78707300631

13. Plebani M, Laposata M, Lundberg GD. The braintobrain loop concept for laboratory testing 40 years after its introduction. Am J Clin Pathol 2011; 136(6):829-833. doi: 10.1309/ AJCPR28 HWH SSD NON.

14. Boone DJ. Governmental perspectives on evaluating laboratory performance. Clin Chem. 1993;39(7):14611467.

15. Bonini P, Plebani M, Ceriotti F. Errors in laboratory medicine. Clin Chem. 2002;48(6):691-698.

16. Carraro P, Plebani M. Errors in a stat laboratory: Types and frequencies 10 years later. Clin Chem. 2007; 53(7):1338-1342. doi: 10.1373/clinchem.2007.088344

\section{How to cite this article?}

Patel K. Preanalytical issues identification and prevention in clinical hematology laboratory: best practice for patient safety. Trop J Path Micro 2019;5(9):619-626.doi:10.17511/jopm.2019.i09.02. 